\title{
Comment désirez-vous mourir?
}

\section{Hans Stalder}

Prof. Dr med., membre de la rédaction

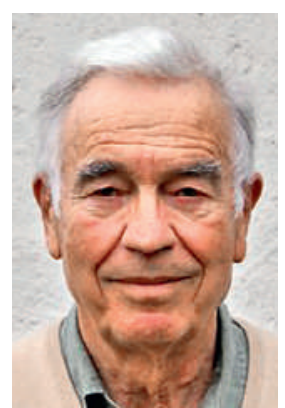

Dans son Et encore... du 25 novembre 2015, intitulé Plutôt jeune, riche et en bonne santé [1], Anna Sax nous communique son espérance de vie calculée selon un questionnaire facilement disponible sur internet: Anna devrait atteindre 98 ans! J’ai fait la même chose; quelle déception: je n'arrive qu'à 94 ans... En trichant un peu - j'ai changé mon sexe dans le formulaire - j'arrive presqu'à l'âge d'Anna. Aurait-elle dû appeler son éditorial Plutôt jeune, riche, en bonne santé et de sexe féminin? Peut-être pas, car si la durée de vie des femmes est plus longue que celle des hommes, elle est associée à davantage de maladies ou en tout cas à plus de consultations médicales. De plus, Anna Sax elle-même reste un peu perplexe de devoir vivre encore une fois plus de 40 ans dans ce monde [1].

\section{Les étiologies cardiaques et tumorales ont fortement diminué cette dernière décennie, et la démence a presque triplé.}

Si l'on pose dans son entourage la question: «Comment voulez-vous mourir?», on vous répond en général soit immédiatement, soit après réflexion, mais avec dignité: surtout pas dans un état de démence en perdant son autonomie. Or, comme le montre l'Office fédéral de la statistique [2], les causes de mort et par conséquent la vie de nos dernières années sont en train de changer: les étiologies cardiaques et tumorales ont fortement diminué cette dernière décennie, et la démence a presque triplé. Et rien ne nous prédit que cela ne va pas continuer, car actuellement nous faisons tout pour que cela arrive. La politique de santé encourage fortement le dépistage et les limites d'âge pour telle ou telle intervention sont de plus en plus repoussées [3]. Même si les dépistages n'augmentent souvent pas la survie [4], ils tendent à diminuer l'incidence des cancers et des infarctus sans que l'on sache quelle sera la qualité des jours gagnés et comment sera la mort sans ces maladies. Logiquement, la prévalence des démences devrait augmenter et cela malgré le fait que son incidence semble diminuer. Et il n'est pas utile ici d'introduire la notion des quality adjusted life years (QALYs) ajoutés, car les personnes démentes ne sont guère capables d'estimer leur qualité de vie ou, si elles le sont, de nous le transmettre sur un formulaire. Un autre exemple (pour ceux qui aimeraient mourir subi- tement): on pose des défibrillateurs partout dans les espaces publics afin de sauver des vies. Même si la survie moyenne après hospitalisation n'est que de 10\% [5] - dans certaines statistiques, avec une méthodologie plus sophistiquée, on arrive à des résultats un peu meilleurs -, ceux qui sortent vivants de l'hôpital ont une bonne chance de souffrir de problèmes neurologiques, voire de démence. Une estimation de la qualité de vie des survivants fait par ailleurs cruellement défaut. Et n'est-il pas aberrant que si nous sommes alors atteints de démence certains gériatres veuillent encore prolonger cet état lamentable par des médicaments? Alors que la mort ne devrait pas être une affaire médicale [6], le paradoxe est qu'elle survient le plus souvent à l'hôpital et que les dernières semaines coûtent très cher à notre système de santé. C'est comme si la mort n'était pas une chose normale, mais une maladie gravissime qui demande un traitement. Or elle demande surtout des soins. Et ces soins devraient plutôt être gérés par des infirmières que par des médecins, parce que eux risquent de nous subtiliser notre autonomie quand nous aimerions finir nos jours [7], car bienveillance n'est pas toujours bienfaisance.

\section{C'est comme si la mort n'était pas une chose} normale, mais une maladie gravissime qui demande un traitement.

N'oublions pas, même si c'est difficile, de demander à nos patients âgés, lorsque se pose la question de la prévention ou des soins des derniers jours: «Comment désirez-vous mourir?»

\section{Références}

1 Sax A. Plutôt jeune, riche et en bonne santé... BMS 2015;96:1790.

2 http://www.statistique.admin.ch/

3 Münzer T. Gesundheitsprävention im Alter - Wer gut beraten wird, lebt länger! Swiss med Forum 2016;16:7-8.

4 Saquib N, Saquib J, Ioannidis JP. Does screening for disease save lives in asymptomatic adults? Systematic review of metaanalyses and randomized trials. Int J Epidemiol. 2015;44:264-77.

5 Savastano S, Klersy C, Raimondi M, Langord K, Vanni V, Rordorf R, Vicentini A, Petracci B, Landolina M, Visconti LO. Positive trend in survival to hospital discharge after out-of-hospital cardiac arrest: a quantitative review of the literature. J Cardiovasc Med 2014;15:609-15.

6 Berner D. A propos de la récente polémique sur l'assistance au suicide. BMS 2015;96:1930.

7 Borasio GD. Point de vue médical sur le suicide assisté la bienveillance négligée. BMS 2015;96:889-91. 